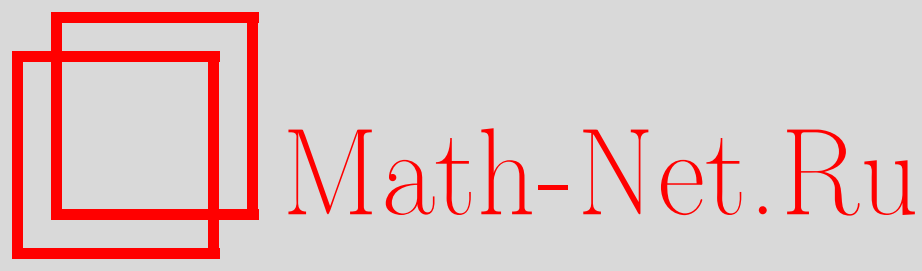

В. В. Киселев, А. А. Расковалов, Взаимодействие бризера с волной намагниченности в ферромагнетике с анизотропией типа “легкая ось", ТМФ, 2010, том 163, номер 1, 94-113

DOI: https://doi.org/10.4213/tmf6489

Использование Общероссийского математического портала Math-Net.Ru подразумевает, что вы прочитали и согласны с пользовательским соглашением http://www.mathnet.ru/rus/agreement

Параметры загрузки:

IP : 3.82 .47 .9

26 апреля 2023 г., 15:54:25

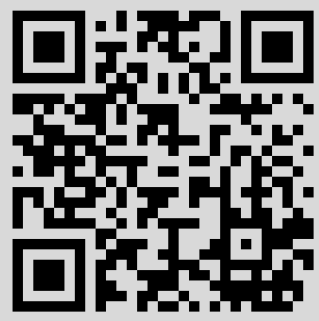




\section{ВЗАИМОДЕЙСТВИЕ БРИЗЕРА С ВОЛНОЙ НАМАГНИЧЕННОСТИ В ФЕРРОМАГНЕТИКЕ С АНИЗОТРОПИЕЙ ТИПА “ЛЕГКАЯ ОСЬ"}

Методом "одевания" найдены точные решения уравнения Ландау-Лифшица для ферромагнетика с анизотропией типа “легкая ось”, которые описывают взаимодействие нелинейной волны прецессии произвольной амплитуды с солитонами. Проанализировано изменение внутренней структуры и физических параметров солитонов в результате их взаимодействия с волной намагниченности. Найдена бесконечная серия интегралов движения, стабилизирующих солитоны на фоне волны накачки.

Ключевые слова: бризер, доменная граница, нелинейная волна намагниченности, уравнение Ландау-Лифшица, задача Римана, метод "одевания".

\section{1. ВВЕДЕНИЕ}

В безразмерных переменных уравнение квазиодномерной динамики ферромагнетика с кристаллографической магнитной анизотропией типа “легкая ось” имеет вид

$$
\partial_{t} \mathbf{S}=\left[\mathbf{S} \times \partial_{x}^{2} \mathbf{S}\right]+\beta^{2}(\mathbf{n} \cdot \mathbf{S})[\mathbf{S} \times \mathbf{n}], \quad \mathbf{S}^{2}=1,
$$

где векторный параметр порядка $\mathbf{S}$ описывает намагниченность среды, $\beta^{2}>0$ постоянная анизотропии, вектор $\mathbf{n}=(0,0,1)$ задает направление оси анизотропии, $x, t-$ пространственная координата и время.

Модель (1.1) является вполне интегрируемой, и к настоящему времени классифицированы все ее солитонные решения с однородной асимптотикой на бесконечности [1]. Они делятся на два типа: доменные стенки и бризеры. Бризеры при определенных условиях можно трактовать как уединенные домены - "зародыши" перемагничивания материала [2]. В легкоосном ферромагнетике изолированная доменная стенка может быть только неподвижной. В работах [3], [4] с теоретической точки зрения описано взаимодействие доменной стенки с бегущей волной намагниченности и получен неожиданный результат: под действием волны доменная стенка начинает двигаться навстречу волне.

* Институт физики металлов УрО РАН, Екатеринбург, Россия. E-mail: kiseliev@imp.uran.ru 
Рассмотрение взаимодействия бризеров и, в частности, уединенных доменов с нелокализованными волнами намагниченности до сих пор проведено не было. Это связано со значительными трудностями, обусловленными существенной нелинейностью модели и наличием нетривиального неоднородного фона. Теоретическое описание нелинейных возбуждений на пространственно нелокализованном фоне в настоящее время возможно только на основе модификаций метода обратной задачи рассеяния. В нашей работе методом “одевания" [5] мы ищем точные решения нелинейной неоднородной краевой задачи, которые описывают взаимодействие нелокализованной волны прецессии произвольной амплитуды с бризерами. Особенность “одевания" состоит в том, что, хотя $U-V$ пара для уравнения (1.1) рациональным образом зависит от спектрального параметра, задача сопряжения аналитических функций, лежащая в основе метода, формулируется не в комплексной плоскости, а на римановой поверхности, которая топологически эквивалента тору и естественным образом связана с волной прецессии. В данном случае преимущество "одевания" перед методом конечнозонного интегрирования [6], [7] состоит в том, что окончательные формулы для бризера и доменной границы на фоне волны выражаются не через многомерные тета-функции, а с помощью хорошо изученных и табулированных эллиптических функций Якоби.

В настоящей работе мы анализируем изменения физических параметров солитонов и их внутренней структуры в результате взаимодействия с волной. Показано, что под действием волны уединенный домен, как и отдельная доменная граница, движется навстречу волне. Найдена бесконечная серия законов сохранения, гарантирующая динамическую стабильность бризера на фоне волны. При наличии волны сохраняющиеся величины зависят не только от данных дискретного спектра, которыми параметризуется бризер в ее отсутствие, но и от характеристик самой волны ее волнового числа и амплитуды. Это обстоятельство следует учитывать при анализе физических свойств солитонов. Найдены условия, при которых нелинейная волна намагниченности разрушает солитоны.

\section{2. НЕЛИНЕЙНЫЕ ВОЗБУЖДЕНИЯ НА ФОНЕ ВОЛНЫ НАМАГНИЧЕННОСТИ: МЕТОД “ОДЕВАНИЯ"}

Нас интересуют решения модели (1.1), описывающие локализованные возбуждения на фоне бегущей волны намагниченности,

$$
\mathbf{S} \rightarrow \mathbf{S}_{ \pm}^{(0)}=\left(\sin \theta_{0} \cos \varphi_{ \pm}, \sin \theta_{0} \sin \varphi_{ \pm}, \cos \theta_{0}\right), \quad x \rightarrow \pm \infty
$$

которая сама является точным решением модели (1.1). В формуле (2.1) угол $\theta_{0}$ задает амплитуду волны, $\varphi_{ \pm}=p x-\omega t+\delta_{ \pm}, \omega=\left(p^{2}+\beta^{2}\right) \cos \theta_{0}-$ частота прецессии, $p-$ волновое число, $\delta_{ \pm}=$const. Далее будет показано, что фазовые сдвиги $\delta_{ \pm}$не могут быть произвольными. Они несут информацию о пространственно локализованных бризерах, которые модулируют волну (2.1). 
Основой метода интегрирования модели (1.1) служит вспомогательная система линейных уравнений

$$
\begin{aligned}
\partial_{x} \chi= & -i\left[w_{1}\left(S_{1} \sigma_{1}+S_{2} \sigma_{2}\right)+w_{3} S_{3} \sigma_{3}\right] \chi \equiv U \chi, \\
\partial_{t} \chi= & -i\left\{w_{1}\left(\left[\mathbf{S} \times \partial_{x} \mathbf{S}\right]_{1} \sigma_{1}+\left[\mathbf{S} \times \partial_{x} \mathbf{S}\right]_{2} \sigma_{2}\right)+w_{3}\left[\mathbf{S} \times \partial_{x} \mathbf{S}\right]_{3} \sigma_{3}-\right. \\
& \left.-2 w_{1}^{2} S_{3} \sigma_{3}-2 w_{1} w_{3}\left(S_{1} \sigma_{1}+S_{2} \sigma_{2}\right)\right\} \chi \equiv V \chi
\end{aligned}
$$

условие совместности которой равносильно уравнению (1.1). Здесь $\sigma_{i}-$ матрицы Паули, коэффициенты $w_{1,3}$ являются функциями спектрального параметра $\lambda$ : $w_{1}=\beta\left(\lambda+\lambda^{-1}\right) / 4, w_{3}=\beta\left(\lambda-\lambda^{-1}\right) / 4$.

Фундаментальные решения системы (2.2), соответствующие граничным распределениям намагниченности $\mathbf{S}_{ \pm}^{(0)}$, имеют вид

$$
\chi_{2,1}^{(0)}(\lambda, \tau, x, t)=\alpha(\lambda, \tau) e^{-i \sigma_{3} \varphi_{ \pm} / 2}\left(\begin{array}{cc}
1 & -\Delta(\lambda, \tau) \\
\Delta(\lambda, \tau) & 1
\end{array}\right) e^{\tau \sigma_{3} \eta / i}
$$

где $\alpha(\lambda, \tau)$ - общий множитель,

$$
\begin{gathered}
\Delta(\lambda, \tau)=\frac{\tau-\left(w_{3} \cos \theta_{0}-p / 2\right)}{w_{1} \sin \theta_{0}}=\frac{w_{1} \sin \theta_{0}}{\tau+w_{3} \cos \theta_{0}-p / 2}, \\
\tau=\left[\left(w_{3} \cos \theta_{0}-\frac{p}{2}\right)^{2}+w_{1}^{2} \sin ^{2} \theta_{0}\right]^{1 / 2}, \quad \eta=x-\left(2 w_{3}+p \cos \theta_{0}\right) t .
\end{gathered}
$$

Для задачи о взаимодействии локализованных возбуждений со спиновой волной определим функции Йоста $\chi_{1,2}$ как решения системы (2.2) с асимптотическим поведением $\chi_{1,2} \rightarrow \chi_{1,2}^{(0)}$ при $x \rightarrow \mp \infty$. Аналитические свойства решений Йоста формулируются на римановой поверхности функции $\tau=\tau(\lambda)$. Для упрощения анализа униформизуем алгебраическую кривую $\tau=\tau(\lambda)$ функциями Якоби [8]:

$$
\begin{aligned}
& \lambda=\left(k^{\prime}\right)^{-1 / 2} \operatorname{tg} \frac{\theta_{0}+\theta_{4}}{2}\left[\frac{i \operatorname{dn}\left(u+i K^{\prime}\right)+k^{\prime} \operatorname{ctg}\left(\left(\theta_{0}+\theta_{4}\right) / 2\right)}{i \operatorname{dn}\left(u+i K^{\prime}\right)-\operatorname{tg}\left(\left(\theta_{0}+\theta_{4}\right) / 2\right)}\right], \\
& \tau=\frac{i \beta k^{2} \sin \theta_{0}\left[\sin \left(\theta_{0}-\theta_{4}\right) \sin \left(\theta_{0}+\theta_{4}\right)\right]^{-1 / 2} \operatorname{cn}\left(u+i K^{\prime}\right) \operatorname{sn}\left(u+i K^{\prime}\right)}{\left[i \operatorname{dn}\left(u+i K^{\prime}\right)+k^{\prime} \operatorname{ctg}\left(\left(\theta_{0}+\theta_{4}\right) / 2\right)\right]\left[i \operatorname{dn}\left(u+i K^{\prime}\right)-\operatorname{tg}\left(\left(\theta_{0}+\theta_{4}\right) / 2\right)\right]} .
\end{aligned}
$$

Здесь $0 \leqslant \theta_{4} \leqslant \theta_{0}$ определяется уравнением $\operatorname{tg} \theta_{4}=\kappa\left(1+\kappa^{2}\right)^{-1 / 2} \operatorname{tg} \theta_{0}, \kappa=p / \beta$, а модуль $k$ и дополнительный модуль $k^{\prime}$ эллиптических функций имеют вид

$$
k=\frac{2 \sqrt{\cos \theta_{0} \cos \theta_{4}}}{\cos \theta_{0}+\cos \theta_{4}}, \quad k^{\prime}=\operatorname{tg} \frac{\theta_{0}+\theta_{4}}{2} \operatorname{tg} \frac{\theta_{0}-\theta_{4}}{2} .
$$

В плоскости комплексной переменной $u$ функции $\lambda=\lambda(u)$ и $\tau=\tau(u)$ являются мероморфными двоякопериодическими функциями с периодами $2 K, 4 i K^{\prime}$, где $K=K(k), K^{\prime}=K\left(k^{\prime}\right)$ - полные эллиптические интегралы первого рода. 


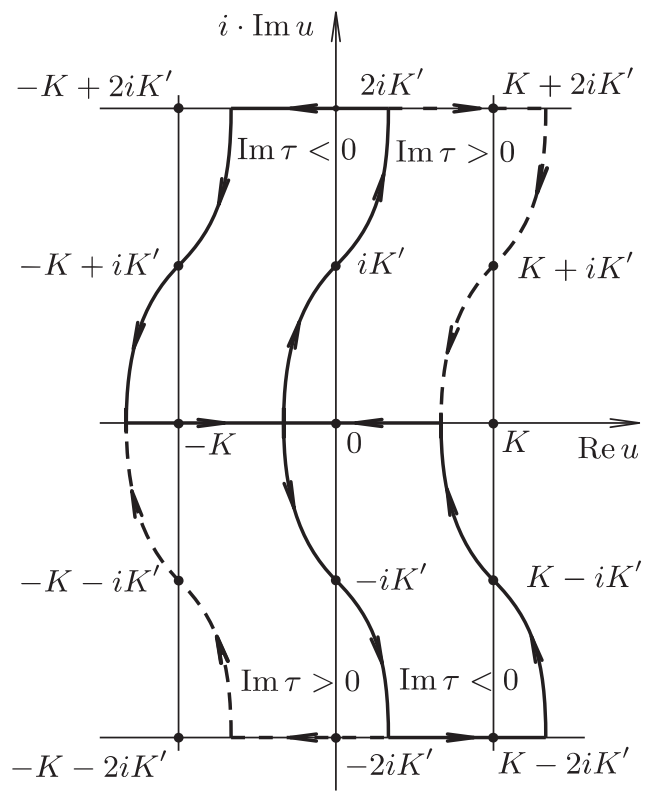

Рис. 1. Области аналитичности решений Йоста. Сплошная линия - контур $\Gamma$, слева от которого $\operatorname{Im} \tau<0$. Штриховая линия - контур, эквивалентный контуру $Г$, справа от которого $\operatorname{Im} \tau>0$.

Выберем множитель $\alpha(\lambda, \tau)$ в формуле (2.3) так, чтобы в терминах нового спектрального параметра $u$ затравочные решения $\chi_{1,2}^{(0)}$ имели наиболее простой вид:

$$
\chi_{1,2}^{(0)}=e^{-i \sigma_{3} \varphi_{\mp} / 2}\left(\begin{array}{cc}
\beta(-u) & -\beta(u) \\
\beta(u) & \beta(-u)
\end{array}\right) e^{\tau \sigma_{3} \eta / i},
$$

где

$$
\beta(u)=\left.\frac{1}{2}\left(\frac{\operatorname{cn} v}{\operatorname{sn} v}-k^{\prime} \frac{\operatorname{sn} v}{\operatorname{cn} v}\right)\right|_{v=\left(u+i K^{\prime}+K / 2\right) / 2} .
$$

Решения Йоста с осциллирующими асимптотиками при $x \rightarrow \pm \infty$ существуют лишь тогда, когда параметр $u$ принимает значения на контуре $\Gamma=\{u: \operatorname{Im} \tau=0\}$, который разделяет области $\Gamma_{+}=\{u: \operatorname{Im} \tau>0\}$ и $\Gamma_{-}=\{u: \operatorname{Im} \tau<0\}$ (см. рис. 1). Множество $\Gamma$ соответствует непрерывному спектру задачи рассеяния. Фундаментальные решения $\chi_{1}(u)$ и $\chi_{2}(u)$ на контуре $\Gamma$ связаны между собой матрицей перехода $T(u)$ :

$$
\chi_{1}(u)=\chi_{2}(u) T(u) .
$$

Вследствие симметрии уравнений (2.2) и функций (2.4) решения Йоста удовлетворяют редукциям

$$
\begin{gathered}
\chi_{1,2}\left(-u+K+2 i K^{\prime}\right)=-\sigma_{3} \chi_{1,2}(u) \sigma_{3}, \quad \chi_{1,2}^{*}\left[\left(-u+2 i K^{\prime}\right)^{*}\right]=-i \sigma_{2} \chi_{1,2}(u), \\
\chi_{1,2}^{*}\left(u^{*}\right)=\frac{\left(1+k^{\prime}\right) \sqrt{k^{\prime}}}{2 \beta(u) \beta(-u)} \sigma_{2} \chi_{1,2}(u) \sigma_{2} .
\end{gathered}
$$

4 Теоретическая и математическая физика, т. 163, № 1, 2010 г. 
Эти соотношения позволяют конкретизировать вид матрицы перехода:

$$
T(u)=\left(\begin{array}{cc}
a(u) & -b^{*}\left(u^{*}\right) \\
b(u) & a^{*}\left(u^{*}\right)
\end{array}\right)
$$

где

$$
\begin{array}{rlrl}
a(u) a^{*}\left(u^{*}\right)+b(u) b^{*}\left(u^{*}\right)=1, & \\
a\left(-u+K+2 i K^{\prime}\right) & =a(u), & b\left(-u+K+2 i K^{\prime}\right) & =-b(u), \\
a^{*}\left[\left(-u+2 i K^{\prime}\right)^{*}\right] & =a(u), & b^{*}\left[\left(-u+2 i K^{\prime}\right)^{*}\right] & =b(u) .
\end{array}
$$

Если $\mathbf{S}(x, t)$ достаточно быстро (например, быстрее любой степени $x$ ) стремится к асимптотическим значениям $\mathbf{S}_{ \pm}^{(0)}(x, t)$, полезно ввести новые матричные функции $\chi_{+}=\left(\chi_{1}^{(1)}, \chi_{2}^{(2)}\right)$ и $\chi_{-}=\left(\chi_{2}^{(1)}, \chi_{1}^{(2)}\right)$, которые допускают аналитическое продолжение с контура $Г$ в области $\Gamma_{+}$и $\Gamma_{-}$соответственно. Здесь $\chi^{(i)}$ означает $i$-й столбец матричной функции $\chi(x, t, u)$.

Из соотношения (2.5) получаем представления для элементов матрицы перехода:

$$
a(u)=\frac{\operatorname{det} \chi_{+}(u)}{\operatorname{det} \chi_{2}^{(0)}(u)}, \quad a^{*}\left(u^{*}\right)=\frac{\operatorname{det} \chi_{-}(u)}{\operatorname{det} \chi_{2}^{(0)}(u)} .
$$

Отсюда следует, что функции $a(u)$ и $a^{*}\left(u^{*}\right)$ аналитически продолжаются с контура $Г$ в области $\Gamma_{+}$и $\Gamma_{-}$соответственно (за исключением, быть может, точек, где $\left.\operatorname{det} \chi_{2}^{(0)}(u)=\beta^{2}(-u)+\beta^{2}(u)=0\right)$.

Множество точек, в которых функции $a(u)$ и $a^{*}\left(u^{*}\right)$ обращаются в нуль, отвечает дискретному спектру линейной системы (2.2). В силу редукций $(2.7)$ функция $a^{*}\left(u^{*}\right)$ в области $\Gamma_{-}$имеет два особых нуля

$$
u=i K^{\prime}-\frac{K}{2}, \quad u=-i K^{\prime}+\frac{K}{2} \quad\left(\bmod \left(2 K, 4 i K^{\prime}\right)\right)
$$

и множество нулей вида

$$
\begin{array}{ll}
u=\mu_{s}, & u=-\mu_{s}^{*}+2 i K^{\prime}, \\
u=\mu_{s}^{*}+K, & u=-\mu_{s}-K-2 i K^{\prime} \quad\left(\bmod \left(2 K, 4 i K^{\prime}\right)\right),
\end{array}
$$

где $\mu_{s} \in \Gamma_{-}-$комплексные параметры, $s=1,2, \ldots$. Особые нули (2.9) приводят к появлению доменной стенки, в то время как каждая группа нулей вида (2.10) учитывает наличие бризера на фоне волны (2.1). Каждая доменная стенка связывает разные равновесные состояния намагниченности. В нашей задаче в силу краевых условий (2.1) доменные стенки либо отсутствуют, либо появляются парами с противоположными топологическими зарядами. Другими словами, в данной задаче особые нули (2.9) или отсутствуют, или являются кратными. Напомним, что кратные нули приводят к появлению слабо связанных экспоненциально-полиномиальных солитонов [9]. Далее для определенности будем предполагать, что нули функций $a(u)$ и $a^{*}\left(u^{*}\right)$ являются простыми, тогда особые нули (2.9) можно не учитывать. 
Для дальнейшего анализа удобно выделить из функций $\chi_{ \pm}$затравочное решение $\chi_{2}^{(0)}$ и ввести матричные функции $\Psi_{ \pm}$с помощью соотношений

$$
\chi_{-}(u)=\Psi_{-}(u) \chi_{2}^{(0)}(u), \quad \frac{\chi_{+}(u)}{a(u)}=\Psi_{+}(u) \chi_{2}^{(0)}(u) .
$$

Тогда уравнение (2.5) сведется к условию сопряжения аналитических функций $\Psi_{ \pm}$ на контуре Г:

$$
\Psi_{-}(x, t, u)=\Psi_{+}(x, t, u) G(x, t, u), \quad u \in \Gamma,
$$

где

$$
G(x, t, u)=\chi_{2}^{(0)}(x, t, u)\left(\begin{array}{cc}
1 & -b^{*}\left(u^{*}\right) \\
-b(u) & 1
\end{array}\right)\left(\chi_{2}^{(0)}\right)^{-1}(x, t, u) .
$$

Мы пришли к классической задаче Римана на римановой поверхности. В данном случае эта поверхность - тор, т.к. все функции в формуле (2.11) двоякопериодичны по спектральному параметру $u$ с периодами $2 K, 4 i K^{\prime}$ [8], [10]. Отличительная особенность задачи Римана на торе [11]-[13] состоит в том, что структура матричных функций $\Psi_{ \pm}$достаточно жестко задается редукциями (2.6), которые легко переносятся на новые матричные функции $\Psi_{ \pm}$. В областях своей аналитичности функции $\Psi_{ \pm}$удовлетворяют ограничениям

$$
\Psi_{ \pm}\left(-u+K+2 i K^{\prime}\right)=\sigma_{3} \Psi_{ \pm}(u) \sigma_{3}, \quad \Psi_{ \pm}^{*}\left[\left(-u+2 i K^{\prime}\right)^{*}\right]=\sigma_{2} \Psi_{ \pm}(u) \sigma_{2}
$$

а на контуре Г одну из них можно выразить через другую:

$$
\Psi_{+}^{-1}(u)=\Psi_{-}^{\dagger}\left(u^{*}\right), \quad u \in \Gamma
$$

(здесь и далее верхний индекс “†” означает эрмитово сопряжение). В итоге условие на контуре Г можно записать в терминах одной лишь функции $\Psi_{-}$:

$$
\Psi_{-}^{\dagger}\left(u^{*}\right) \Psi_{-}(u)=G(u)
$$

Получим формулу для расчета намагниченности. Пусть $0<u_{0}<K$ - вещественный корень уравнения $i \operatorname{dn}\left(u_{0}+i K^{\prime}\right)=\operatorname{tg}\left(\left(\theta_{0}+\theta_{4}\right) / 2\right)$. В точке $u=u_{0}$ функция $\tau(u)$ имеет простой полюс, а правая часть формулы (2.13) - существенную особенность. С другой стороны, левая часть равенства (2.13) в малой окрестности точки $u=u_{0}$ является функцией, дифференцируемой по параметру $u$. Противоречия можно избежать лишь при условии, что $b\left(u_{0}\right)=0$ или, эквивалентно, при условии, что матричная функция $\Psi_{-}(u)$ унитарна в точке $u=u_{0}$ :

$$
\Psi_{-}^{\dagger}\left(u_{0}\right) \Psi_{-}\left(u_{0}\right)=I
$$

Функция $\Psi_{-}$удовлетворяет дифференциальным уравнениям

$$
\partial_{x} \Psi_{-}=U \Psi_{-}-\Psi_{-} U_{0}, \quad \partial_{t} \Psi_{-}=V \Psi_{-}-\Psi_{-} V_{0},
$$

где матричные функции $U, V$ определены формулами (2.2), $U_{0}$ и $V_{0}$ - значения $U$ и $V$ при $\mathbf{S}=\mathbf{S}_{+}^{(0)}$. Отсюда, в частности, получаем равенство $U=\partial_{x} \Psi_{-} \Psi_{-}^{-1}+\Psi_{-} U_{0} \Psi_{-}^{-1}$, 
левая и правая части которого имеют простые полюсы при $u=u_{0}$. Приравнивая вычеты в этой точке и учитывая (2.14), получаем формулу для расчета намагниченности:

$$
S_{k} \sigma_{k}=\Psi_{-}\left(u_{0}\right)\left(\mathbf{S}_{+}^{(0)}\right)_{k} \sigma_{k} \Psi_{-}^{\dagger}\left(u_{0}\right) .
$$

Для того чтобы ей воспользоваться, нужно знать решения задачи Римана (2.11) на торе. Эти решения в зависимости от вида коэффициентов $a(u), b(u), a^{*}\left(u^{*}\right), b^{*}\left(u^{*}\right)$ подразделяются на два типа.

Первый тип решений соответствует регулярной задаче Римана, когда функции $b(u), b^{*}\left(u^{*}\right)$ отличны от нуля на контуре $\Gamma$, а функции $a(u), a^{*}\left(u^{*}\right)$ не имеют нулей в областях своей аналитичности. Решения регулярной задачи Римана определяются из системы линейных интегральных уравнений на торе [11], [12] и описывают локализованные возбуждения на фоне волны (2.1), которые расплываются со временем из-за эффектов дисперсии.

Второй тип решений представляет особый интерес. Он описывает солитоноподобные возбуждения, которые движутся подобно частицам на фоне волны (2.1) и восстанавливают свою внутреннюю структуру после столкновений друг с другом. Солитонные состояния соответствуют решениям задачи Римана (2.11) с нулями коэффициентов $a$ и $a^{*}$ в областях $\Gamma_{+}$и $\Gamma_{-}$, когда $b=b^{*} \equiv 0$. В этом случае функции $\Psi_{-}(u), \Psi_{-}^{\dagger}\left(u^{*}\right)$ оказываются мероморфными в области $\Gamma_{-} \cup \Gamma_{+}$. Условие их сопряжения на контуре Г приобретает простой вид

$$
\Psi_{-}^{\dagger}\left(u^{*}\right) \Psi_{-}(u)=\Psi_{-}(u) \Psi_{-}^{\dagger}\left(u^{*}\right)=I, \quad u \in \Gamma .
$$

В силу соотношений (2.8) и (2.17) нули функции $\Psi_{-}(u)$ совпадают с нулями коэффициента $a^{*}\left(u^{*}\right)$ и являются полюсами для функции $\Psi_{-}^{\dagger}\left(u^{*}\right)$.

Построим простейшую солитонную функцию $\Psi_{-}^{\dagger}\left(u^{*}\right)$, описывающую один бризер на фоне волны (2.1). В силу (2.10) такая функция имеет четыре простых полюса, поэтому ее разложение по дзета-функциям Вейерштрасса должно иметь вид [10]

$$
\Psi_{-}^{\dagger}\left(u^{*}\right)=A_{0}+A \zeta_{1}+B \zeta_{2}+C \zeta_{3}+D \zeta_{4}
$$

где $\zeta_{i}=\zeta\left(u-\mu_{i}\right), \mu_{1}=\mu, \mu_{2}=-\mu-K-2 i K, \mu_{3}=-\mu^{*}+2 i K^{\prime}, \mu_{4}=\mu^{*}+K, \mu \in \Gamma_{-}$, периоды функций Вейерштрасса суть $2 K, 4 i K^{\prime}$. Учитывая трансформационные свойства функций Вейерштрасса [8], [10], из условий симметрии (2.12) выражаем матрицы $B, C, D$ через $A$ :

$$
B=-\sigma_{3} A \sigma_{3}, \quad C=-\sigma_{2} A^{*} \sigma_{2}, \quad D=\sigma_{1} A^{*} \sigma_{1} .
$$

При этом матрица $A_{0}$ имеет вид

$$
A_{0} \equiv\left(\begin{array}{cc}
a_{0} & 2 \eta_{2} A_{12} \\
2 \eta_{2} A_{21} & a_{0}^{*}
\end{array}\right),
$$

где $\eta_{2}=\zeta\left(-K-2 i K^{\prime}\right)$. Матричная функция $(2.18)$ должна иметь периоды $2 K$, $4 i K^{\prime}$. Это возможно, только если сумма вычетов функции $\Psi_{-}^{\dagger}\left(u^{*}\right)$ в ее полюсах $\mu_{i}$ 
равна нулю [10]. Данное условие будет выполнено, если недиагональные элементы матрицы $A$ удовлетворяют ограничению

$$
A_{12}=-A_{21}^{*}
$$

Дальнейшие вычисления проведем, предполагая, что это условие выполнено. Окончательный результат подтвердит правильность такого предположения [11]-[13].

Редукции конкретизировали алгебраическую структуру функции (2.18). Положим $A=\tilde{A} R$, где $R=\operatorname{diag}\left(r, r^{*}\right), r \neq 0$, и представим полученный результат в форме, удобной для дальнейшего анализа:

$$
\begin{aligned}
\Psi_{-}^{\dagger}\left(u^{*}\right) & =\Psi^{\dagger}\left(u^{*}\right) R \\
\Psi^{\dagger}\left(u^{*}\right) & =I+\left(\begin{array}{cc}
\tilde{A}_{11} \zeta_{12}(u)-\tilde{A}_{22}^{*} \zeta_{34}(u) & \tilde{A}_{12} \varphi(u) \\
\tilde{A}_{21} \varphi(u) & \tilde{A}_{22} \zeta_{12}(u)-\tilde{A}_{11}^{*} \zeta_{34}(u)
\end{array}\right),
\end{aligned}
$$

где

$$
\zeta_{12}(u)=\zeta_{1}-\zeta_{2}, \quad \zeta_{34}(u)=\zeta_{3}-\zeta_{4}, \quad \varphi(u)=\zeta_{1}+\zeta_{2}-\zeta_{3}-\zeta_{4}+2 \eta_{2} .
$$

Условия отсутствия полюсов в произведении матричных функций

$$
\Psi_{-}(u) \Psi_{-}^{\dagger}\left(u^{*}\right)=R^{\dagger} \Psi(u) \Psi^{\dagger}\left(u^{*}\right) R=I
$$

эквивалентны одному независимому матричному уравнению $\left.\tilde{A}^{\dagger} \Psi^{\dagger}\left(u^{*}\right)\right|_{u=\mu^{*}}=0$, оно имеет нетривиальное решение, только если $\tilde{A}_{\alpha \beta}=m_{\alpha} X_{\beta}, \alpha, \beta=1,2$ [5]. Учитывая это условие и тождество $\varphi\left(\mu^{*}\right)+\zeta_{34}\left(\mu^{*}\right)=-\zeta_{12}^{*}\left(\mu^{*}\right)$, находим

$$
X_{1}=-\frac{m_{1}^{*}}{\left|m_{1}\right|^{2} \zeta_{12}\left(\mu^{*}\right)-\left|m_{2}\right|^{2} \zeta_{12}^{*}\left(\mu^{*}\right)}, \quad X_{2}=\frac{m_{2}^{*}}{\left|m_{1}\right|^{2} \zeta_{12}^{*}\left(\mu^{*}\right)-\left|m_{2}\right|^{2} \zeta_{12}\left(\mu^{*}\right)} .
$$

Построенная матрица $A_{\alpha \beta}$ удовлетворяет ограничению (2.19). Знаменатели, появившиеся в формулах $(2.21)$, можно исключить, переопределяя параметры $r$ и $r^{*}$, тогда матричная функция $\Psi^{\dagger}\left(u^{*}\right)$ примет вид

$$
\Psi^{\dagger}\left(u^{*}\right)=\left(\begin{array}{cc}
-\left|m_{1}\right|^{2} \varphi_{1}(u)-\left|m_{2}\right|^{2} \varphi_{2}(u) & m_{1} m_{2}^{*} \varphi(u) \\
-m_{2} m_{1}^{*} \varphi(u) & \left|m_{1}\right|^{2} \varphi_{2}(u)+\left|m_{2}\right|^{2} \varphi_{1}(u)
\end{array}\right)
$$

где $\varphi_{1}(u)=\zeta_{12}(u)-\zeta_{12}\left(\mu^{*}\right), \varphi_{2}(u)=\zeta_{34}(u)+\zeta_{12}^{*}\left(\mu^{*}\right)$.

Отметим, что функции $\varphi(u), \varphi_{1,2}(u)$ образуют представление группы редукций:

$$
\begin{aligned}
\varphi_{1,2}\left(-u+K+2 i K^{\prime}\right) & =\varphi_{1,2}(u), & \varphi\left(-u+K+2 i K^{\prime}\right) & =-\varphi(u) ; \\
\varphi_{1,2}^{*}\left[\left(-u+2 i K^{\prime}\right)^{*}\right] & =-\varphi_{1,2}(u), & \varphi^{*}\left[\left(-u+2 i K^{\prime}\right)^{*}\right] & =\varphi(u) .
\end{aligned}
$$

Выразим комбинации дзета-функций Вейерштрасса $\varphi_{1,2}(u), \varphi(u)$ в терминах более популярных и табулированных эллиптических функций Якоби с модулем $k$. 
Для сокращения записи введем обозначения $\tilde{\mu}=\mu+i K^{\prime}+K / 2, \tilde{s}=\operatorname{sn}(\tilde{\mu}, k)$, $s=\operatorname{sn}\left(u+i K^{\prime}+K / 2, k\right)$ и аналогичные для других функций Якоби. Тогда

$$
\begin{gathered}
\varphi(u)=-\frac{k^{2}\left(k^{\prime}+|\tilde{d}|^{2}\right) s c}{(d-\tilde{d})\left(k^{\prime}+\tilde{d}^{*} d\right)}, \\
\varphi_{1}(u)=-\frac{k^{2} \tilde{s} \tilde{c}}{\tilde{d}+\tilde{d}^{*}}\left(\frac{d+\tilde{d}^{*}}{d-\tilde{d}}\right), \quad \varphi_{2}(u)=-\frac{k^{2} \tilde{s}^{*} \tilde{c}^{*}}{\tilde{d}+\tilde{d}^{*}}\left(\frac{d \tilde{d}-k^{\prime}}{d \tilde{d}^{*}+k^{\prime}}\right) .
\end{gathered}
$$

Эти и другие тождества настоящей работы проще всего проверить путем разложения их левых и правых частей либо на "простые дроби", либо на "множители" по функциям Вейерштрасса [10]. Константы в подобных разложениях фиксируются или значениями функций в какой-либо точке, или свойствами симметрии, например такими, как (2.23).

При конкретных расчетах используюся тождества

$$
\begin{gathered}
\varphi_{1}^{*}\left(u^{*}\right) \varphi(u)+\varphi^{*}\left(u^{*}\right) \varphi_{2}(u)=0, \\
\varphi_{1}(u) \varphi_{2}^{*}\left(u^{*}\right)+\varphi_{2}(u) \varphi_{1}^{*}\left(u^{*}\right)+\varphi(u) \varphi^{*}(u)=c_{2}, \\
\varphi_{1}^{*}\left(u^{*}\right) \varphi_{1}(u)=\varphi_{2}^{*}\left(u^{*}\right) \varphi_{2}(u)=c_{1}, \\
\varphi^{2}(u)-\varphi_{1}^{2}(u)-\varphi_{2}^{2}(u)=-\frac{c_{2}}{c_{1}} \varphi_{1}(u) \varphi_{2}(u), \\
\beta(u) \beta^{-1}(-u)=\left(k^{\prime}\right)^{-1 / 2} \operatorname{dn}\left(u+\frac{K}{2}\right),
\end{gathered}
$$

где постоянные $c_{1}$ и $c_{2}$ имеют вид

$$
c_{1}=\frac{k^{4}|\tilde{s} \tilde{c}|^{2}}{\left(\tilde{d}+\tilde{d}^{*}\right)^{2}}, \quad c_{2}=\frac{2}{\left(\tilde{d}+\tilde{d}^{*}\right)^{2}}\left[\left(1+k^{\prime}\right)|\tilde{d}|^{2}+\left|k^{\prime}+\tilde{d}\right|^{2}\right] .
$$

Модуль числа $r$ найдем из соотношения (2.20):

$$
\left(R R^{\dagger}\right)^{-1} \equiv|r|^{-2} I=\Psi(u) \Psi^{\dagger}(u)
$$

По построению правая часть равенства не зависит от $u$ и является положительно определенной диагональной матрицей. Непосредственные вычисления с учетом тождеств (2.25) дают

$$
|r|^{-2}=\left(\left|m_{1}\right|^{4}+\left|m_{2}\right|^{4}\right) c_{1}+\left|m_{1} m_{2}\right|^{2} c_{2}
$$

При записи решений модели (1.1) предпочтительнее представление $|r|^{-2}$, которое получается из $(2.26)$, если заменить постоянные $c_{1,2}$ комбинациями из чисел $\varphi_{1,2}\left(u_{0}\right)$ и $\varphi\left(u_{0}\right)($ см. $(2.25))$.

Зависимости вектора $\mathbf{m}=\left(m_{1}, m_{2}\right)$ и фазы $\varphi_{0}=\arg r$ от координат $x, t$ следуют из уравнений, обеспечивающих отсутствие лишних полюсов в выражениях (2.15) для $U$ и $V[5]$. Решениями этих уравнений будут

$$
\mathbf{m}=\chi_{2}^{(0)}(\mu) \mathbf{e}, \quad \varphi_{0}=\text { const }
$$


где $\mathbf{e}=\left(e_{1}, e_{2}\right)$ - произвольный постоянный комплексный вектор.

Непосредственно вычисляя коэффициент $a^{*}\left(u^{*}\right)$,

$$
a^{*}\left(u^{*}\right)=\operatorname{det} \Psi_{-}^{\dagger}\left(u^{*}\right)=-\frac{\varphi_{1}(u) \varphi_{2}(u)}{c_{1}}=\frac{\varphi^{*}\left(u^{*}\right)}{\varphi(u)},
$$

убеждаемся, что в соответствии с предписанными аналитическими свойствами функция $a^{*}\left(u^{*}\right)$ имеет нули в точках $(2.10)$, полюсы в комплексно сопряженных точках и удовлетворяет условию $a^{*}\left(u^{*}\right) a(u)=1$. Для нахождения постоянной $\varphi_{0}$ воспользуемся формулой связи фундаментальных решений на контуре $\Gamma$, которая следует из соотношения (2.5):

$$
\Psi_{-}(u) \chi_{2}^{(0)}(u)=\chi_{2}(u)\left(\begin{array}{cc}
1 & 0 \\
0 & a^{*}\left(u^{*}\right)
\end{array}\right)
$$

Требуя совпадения при $x \rightarrow+\infty$ асимптотик левой и правой частей этого равенства, получаем

$$
\varphi_{0}=i \ln \left(-\frac{\tilde{s}|\tilde{c}|}{\tilde{c}|\tilde{s}|}\right)=\pi-\arg \frac{\tilde{s}}{\tilde{c}}
$$

\section{3. ВЫНУЖДЕННОЕ ДВИЖЕНИЕ УЕДИНЕННОГО ДОМЕНА В ПОЛЕ СПИНОВОЙ ВОЛНЫ}

С помощью формул (2.16), (2.22), (2.26), (2.27), (2.29) находим решение модели (1.1), описывающее бризер на фоне нелинейной спиновой волны (2.1) произвольной амплитуды. Окончательный результат можно записать в виде

$$
\begin{aligned}
S_{3} & =\frac{\left(|\alpha|^{2}-|\beta|^{2}\right) \cos \theta_{0}-\left(\alpha^{*} \beta+\alpha \beta^{*}\right) \sin \theta_{0}}{|\alpha|^{2}+|\beta|^{2}}, \\
S_{1}-i S_{2} & =\frac{e^{-i \varphi_{+}-2 i \varphi_{0}}\left(2 \alpha \beta \cos \theta_{0}+\left(\alpha^{2}-\beta^{2}\right) \sin \theta_{0}\right)}{|\alpha|^{2}+|\beta|^{2}} .
\end{aligned}
$$

Здесь

$$
\begin{gathered}
\alpha=\left|n_{2}\right|^{2} \frac{\varphi_{1}\left(u_{0}\right)}{\varphi\left(u_{0}\right)}-\left|n_{1}\right|^{2} \frac{\varphi_{1}^{*}\left(u_{0}\right)}{\varphi^{*}\left(u_{0}\right)}, \quad \beta=n_{1} n_{2}^{*}, \\
n_{1}=-\left(i \sqrt{k^{\prime}} \frac{\tilde{s}}{\tilde{c}} e^{\gamma}+e^{-\gamma}\right), \quad n_{2}=e^{\gamma}-i \sqrt{k^{\prime}} \frac{\tilde{s}}{\tilde{c}} e^{-\gamma}, \\
\gamma=-i \tau(\mu)\left[x-\left(2 w_{3}(\mu)+p \cos \theta_{0}\right) t\right]+\frac{1}{2} \ln \frac{e_{1}}{e_{2}} .
\end{gathered}
$$

В силу (2.24) коэффициенты $\varphi\left(u_{0}\right)$ и $\varphi_{1}\left(u_{0}\right)$ выражаются в терминах эллиптических функций Якоби от аргументов $\tilde{u}_{0}=u_{0}+i K^{\prime}+K / 2$ и $\tilde{\mu}=\mu+i K^{\prime}+K / 2$. В тех же 
терминах запишем коэффициенты $\tau(\mu)$ и $w_{1,3}(\mu)$ :

$$
\begin{aligned}
\tau(\mu) & =\frac{i \beta k^{2}\left(1-k^{\prime}\right)\left(k^{\prime}-\tilde{d}^{2}\right) s_{0}^{2} c_{0}^{2}}{2\left(k^{\prime}-d_{0}^{2}\right)\left(\tilde{d}-d_{0}\right)\left(k^{\prime}-\tilde{d} d_{0}\right)}, \\
w_{1}(\mu) & =\frac{i \beta k^{2}\left(1-k^{\prime}\right) \tilde{s} \tilde{c} s_{0} c_{0}}{2\left(\tilde{d}-d_{0}\right)\left(k^{\prime}-\tilde{d} d_{0}\right)}, \\
w_{3}(\mu) & =\frac{i \beta}{2\left(1+k^{\prime}\right)\left(\tilde{d}-d_{0}\right)\left(k^{\prime}-\tilde{d} d_{0}\right)}\left[\tilde{d} d_{0}\left(1+k^{\prime}\right)^{2}-\left(k^{\prime}+\tilde{d}^{2}\right)\left(k^{\prime}+d_{0}^{2}\right)\right] .
\end{aligned}
$$

Для сокращения записи мы ввели обозначения $s_{0}=\operatorname{sn} \tilde{u}_{0}, \tilde{s}=\operatorname{sn} \tilde{\mu}$ и т.д.

Согласно найденному решению (3.1), пройдя через бризер, волна намагниченности приобретает дополнительный фазовый сдвиг. Поэтому постоянные $\delta_{ \pm}$в формуле (2.1) оказываются связанными между собой:

$$
\delta_{+}-\delta_{-}=4 \arg \frac{\tilde{s}}{\tilde{c}} \quad(\bmod 2 \pi)
$$

Заметим также, что в силу тождеств

$$
\begin{gathered}
\kappa=\frac{p}{\beta}=\frac{i\left(1-k^{\prime}\right) d_{0}}{k^{\prime}-d_{0}^{2}}, \\
\sin \theta_{0}=\frac{2 i\left(1-k^{\prime}\right) \sqrt{k^{\prime}} s_{0} c_{0}}{k^{\prime}-d_{0}^{2}}, \quad \cos \theta_{0}=\left(\frac{d_{0}^{2}+k^{\prime}}{k^{\prime}-d_{0}^{2}}\right)\left(\frac{1-k^{\prime}}{1+k^{\prime}}\right)
\end{gathered}
$$

в качестве параметров $p$ и $\theta_{0}$ волны (2.1) можно использовать $k^{\prime}$ и $u_{0}$.

Кратко охарактеризуем полученное решение. Ширина бризера (3.1) имеет порядок $-[\operatorname{Im} \tau(\mu)]^{-1}$, а его скорость равна

$$
V=\frac{2 \operatorname{Im}\left[\tau(\mu) w_{3}(\mu)\right]}{\operatorname{Im} \tau(\mu)}+p \cos \theta_{0} .
$$

Бризер представляет собой локализованную модуляцию волны (2.1), которая движется на фоне волны подобно частице. В области локализации солитона происходят обусловленные волной накачки дополнительные пульсации намагниченности с частотой $\Omega=2 \operatorname{Re}\left[\tau(\mu)\left(2 w_{3}(\mu)+p \cos \theta_{0}\right)\right]$ в лабораторной системе координат или с частотой $\omega=\Omega-2 V \operatorname{Re} \tau(\mu)$ в системе отсчета, связанной с бризером. Пульсации распространяются по области локализации бризера, зарождаясь у одного его края и исчезая у другого. Их фазовая скорость равна $V_{\mathrm{ph}}=\Omega /(2 \operatorname{Re} \tau(\mu))$. Величина $[2 \operatorname{Re} \tau(\mu)]^{-1}$ дает характерный масштаб дополнительных пространственных модуляций бризера, связанных с распространением пульсаций.

Бризер существует только в области значений физических параметров задачи, удовлетворяющих неравенству $\operatorname{Im} \tau(\mu)<0$. При увеличении $|\operatorname{Im} \tau(\mu)|$ градиенты огибающей солитона становятся значительными, и периодический рельеф в области локализации солитона становится менее заметен. Когда $\operatorname{Im} \tau(\mu) \rightarrow-0$, область локализации бризера неограниченно увеличивается - волна (2.1) разрушает бризер. При значениях параметра $\mu \in \Gamma_{-}$вблизи наклонных участков контура $Г$ разрушение 
бризера сопровождается генерированием автоколебаний намагниченности. В общем случае картина распределения намагниченности в бризере очень сложна. В пределе $u_{0}=\varepsilon+K / 2, \varepsilon \rightarrow 0, k^{\prime} \rightarrow 0$, при условии $\mu+K / 2=$ const, выражение (3.1) описывает бризер в отсутствие спиновой волны. При конкретных значениях параметра $\tilde{\mu}$ получаем различные солитоны с однородной асимптотикой при $|x| \rightarrow \infty$, которые совпадают с найденными ранее в работе [1] путем непосредственного интегрирования уравнения Ландау-Лифшица.

Обсудим наиболее интересный частный случай общего решения (3.1), который соответствует взаимодействию уединенного домена с нелинейной спиновой волной. С математической точки зрения в этом случае нули (2.10) коэффициента $a^{*}\left(u^{*}\right)$ приближаются к особым значениям (2.9), так что $\tilde{\mu}=2 i K^{\prime}+\varepsilon$. Комплексный параметр $\varepsilon$ определяет внутреннюю структуру и протяженность уединенного домена. В частности, размер домена будет тем больше, чем меньше $|\varepsilon|$. При достаточно малых $|\varepsilon|$ распределение намагниченности (3.1) хорошо аппроксимируется выражением

$$
\begin{aligned}
S_{3} & \approx \tau\left(\cos \theta_{0}\left[\frac{\left|\varepsilon_{0}\right|^{2}}{2} \operatorname{ch} 2 y-1\right]+\frac{\kappa \sin \theta_{0}}{\sqrt{1+\kappa^{2}}}\left[\varepsilon_{0} \operatorname{sh}(y+i \Phi)+\varepsilon_{0}^{*} \operatorname{sh}(y-i \Phi)\right]\right), \\
S_{1}+i S_{2} & \approx \tau e^{i \Phi_{0}}\left(\frac{1}{4} \sin \theta_{0} e^{-i \Phi}\left[\varepsilon_{0}^{2} e^{-2 y}+\left(\varepsilon_{0}^{*}\right)^{2} e^{2 y}\right]-\right. \\
& \left.-\frac{i}{\sqrt{1+\kappa^{2}}}\left[\varepsilon_{0} e^{-y}\left(1+i \kappa \cos \theta_{0}\right)+\varepsilon_{0}^{*} e^{y}\left(1-i \kappa \cos \theta_{0}\right)\right]-\sin \theta_{0} e^{i \Phi}\right),
\end{aligned}
$$

где

$$
\begin{gathered}
\varepsilon_{0}=\left(1+k^{\prime}\right) \varepsilon, \quad \kappa=\frac{p}{\beta}, \quad \Phi \approx \omega t-p x+\arg \frac{e_{1}}{e_{2}}, \quad \omega=\left(p^{2}+\beta^{2}\right) \cos \theta_{0}, \\
\tau^{-1}=\frac{\left|\varepsilon_{0}\right|^{2}}{2} \operatorname{ch} 2 y+1-\frac{i \sin \theta_{0}}{\sqrt{1+\kappa^{2}}}\left[\varepsilon_{0} \operatorname{sh}(y+i \Phi)-\varepsilon_{0}^{*} \operatorname{sh}(y-i \Phi)\right], \\
y \approx-\beta x \cos \theta_{0}-p \beta t \sin ^{2} \theta_{0}+\ln \left|\frac{e_{1}}{e_{2}}\right|, \quad \Phi_{0}=\arg \frac{e_{1}}{e_{2}}+\delta_{+} .
\end{gathered}
$$

Приближенная формула (3.3) получена в результате "сшивания" асимптотик точного решения (3.1) при $|\varepsilon| \ll 1$ в областях $|y|<1$ и $|y|>1$. Вблизи стенок, ограничивающих домен, справедлива оценка $|\varepsilon| e^{|y|}=O(1)(|y|>1,|\varepsilon|<1)$. Поэтому в разложении (3.3) удержаны слагаемые, содержащие малые коэффициенты $O\left(\varepsilon^{2}\right)$ перед $e^{2|y|}$. Условия применимости приближенного решения (3.3),

$$
\frac{\left|\partial_{x} \Phi+p\right|}{|p|} \ll 1, \quad \frac{\left|\partial_{x} y+\beta \cos \theta_{0}\right|}{\beta \cos \theta_{0}} \ll 1,
$$

нарушаются при малых волновых числах $(p \approx 0)$ и при больших углах прецесии $\left(\theta_{0} \approx \pi / 2\right)$ нелинейной спиновой волны $(2.1)$. 


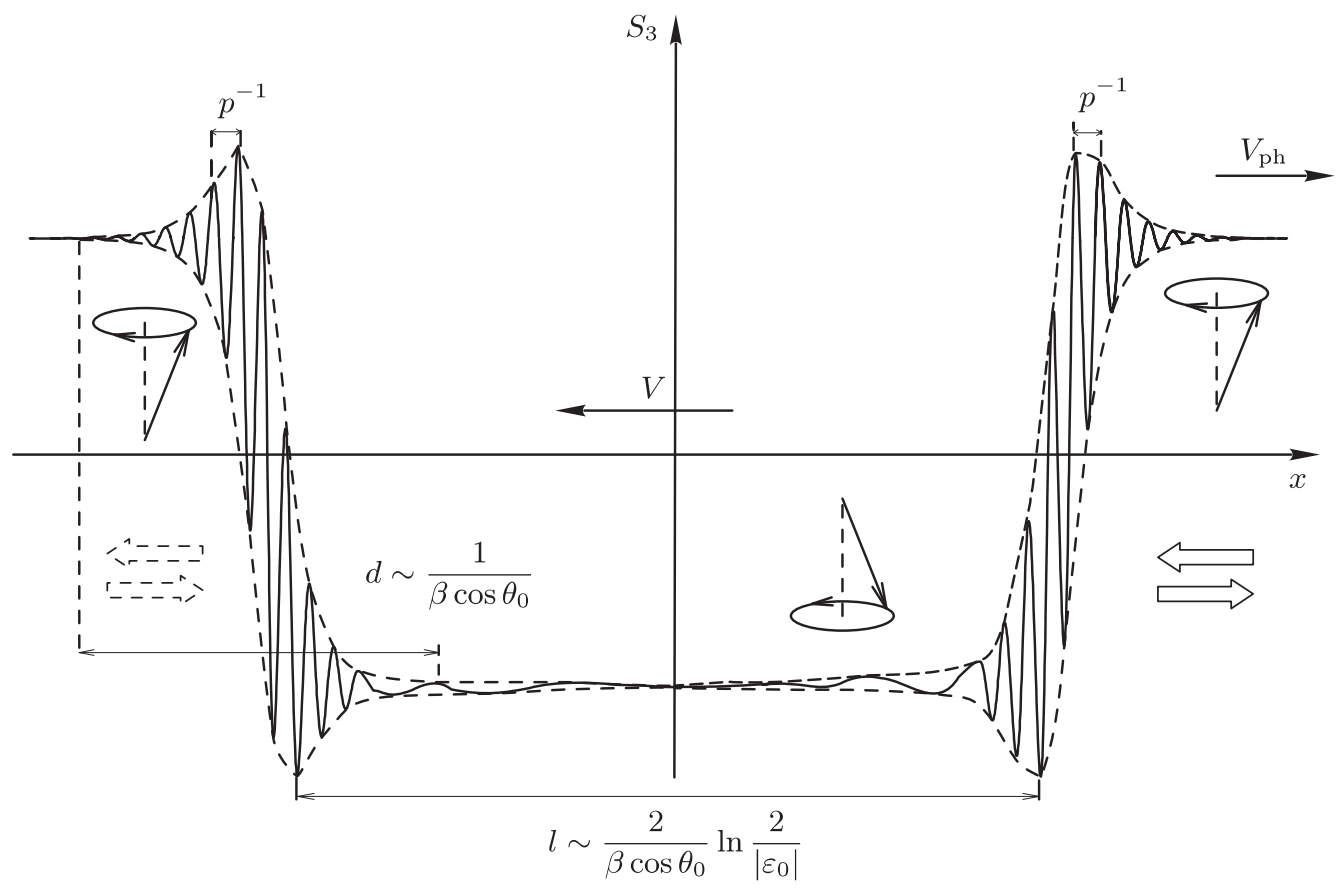

Рис. 2. Вынужденное движение уединенного домена в поле спиновой волны.

Согласно (3.3) в центре уединенного домена (при $y \approx 0)$ распределение намагниченности имеет вид

$$
S_{3} \approx-\cos \theta_{0}, \quad S_{1}+i S_{2} \approx-\sin \theta_{0} \exp \left\{-i\left(p x-\omega t-2 \arg \frac{e_{1}}{e_{2}}-\delta_{+}\right)\right\}
$$

На бесконечности намагниченность совершает неоднородную круговую прецессию вокруг оси $O x_{3}$, связанную с распространением нелинейной спиновой волны:

$$
S_{3} \approx \cos \theta_{0}, \quad S_{1}+i S_{2} \approx \sin \theta_{0} e^{i \varphi_{ \pm}}, \quad x \rightarrow \pm \infty ; \quad \delta_{+}-\delta_{-}=4 \arg \varepsilon_{0} .
$$

Положения стенок, ограничивающих домен, определяются условием $S_{3} \approx 0$. Отсюда находим размер домена: $l \backsim 2\left(\beta \cos \theta_{0}\right)^{-1} \ln \left(2 /\left|\varepsilon_{0}\right|\right)$. Вследствие распространения спиновой волны в пределах доменных стенок намагниченность совершает неоднородную эллиптическую прецессию с нутационными колебаниями оси прецесии. Ширина доменных стенок $d \sim 1 / \beta \cos \theta_{0} \ll l$. Внутри каждой стенки проекция $S_{3}$ резко изменяется от одного из своих квазиравновесных значений $S_{3} \approx \cos \theta_{0}$ до другого $S_{3} \approx-\cos \theta_{0}$. Центры стенок колеблются около средних положений (см. рис. 2 ).

Интересно и важно, что в результате взаимодействия с нелинейной волной уединенный домен начинает двигаться как целое навстречу волне со скоростью $V=-p \sin ^{2} \theta_{0} / \cos \theta_{0}$. 


\section{4. ИНТЕГРАЛЫ ДВИЖКННИЯ ДЛЯ БРИЗЕРА НА ФОНЕ ВОЛНЫ}

В рассматриваемой задаче коэффициент $a^{*}\left(u^{*}\right)$ матрицы перехода $T$ не зависит от времени, поэтому он может использоваться в качестве производящей функции интегралов движения. Удобное для дальнейшего анализа представление коэффициента $a^{*}\left(u^{*}\right)$ находим из формулы $(2.5)$ и условия унимодулярности матрицы $T$ :

$$
a^{*}\left(u^{*}\right)=\lim _{x \rightarrow-\infty}\left[\left(\chi_{1}^{(0)}(x, t, u)\right)^{-1} \chi_{2}(x, t, u)\right]_{11} .
$$

Покажем, что физически содержательные интегралы движения получаются из разложения функции $\ln a^{*}\left(u^{*}\right)$ в асимптотический ряд по степеням $\left(u-u_{0}\right)$. Для этого необходимо найти разложения решения Йоста $\chi_{2}$ по степеням $\left(u-u_{0}\right)$. Представим функцию Йоста $\chi_{2}$ в виде

$$
\chi_{2}=e^{-i \sigma_{3} \varphi_{+} / 2} N[I+\Phi] e^{Z+\tau \sigma_{3} \eta / i}, \quad N=\left(\begin{array}{cc}
\beta(-u) & -\beta(u) \\
\beta(u) & \beta(-u)
\end{array}\right),
$$

где $\Phi$ и $Z$ - соответственно антидиагональная и диагональная матричные функции от переменных $x, t, u$ такие, что $\Phi, Z \rightarrow 0$ при $x \rightarrow+\infty$. Матрица $N$ обладает следующими свойствами:

$$
\begin{gathered}
N^{-1}(u)=N^{\dagger}\left(u^{*}\right) n, \quad n \equiv\left[\beta(u) \beta^{*}\left(u^{*}\right)+\beta(-u) \beta^{*}\left(-u^{*}\right)\right]^{-1}, \\
N^{-1} \sigma_{3} N=\left(w_{3} \cos \theta_{0}-\frac{p}{2}\right) \tau^{-1} \sigma_{3}-w_{1} \sin \theta_{0} \cdot \tau^{-1} \sigma_{1}, \quad N^{-1} \sigma_{2} N=\sigma_{2}, \\
N^{-1} \sigma_{1} N=w_{1} \sin \theta_{0} \cdot \tau^{-1} \sigma_{3}+\left(w_{3} \cos \theta_{0}-\frac{p}{2}\right) \tau^{-1} \sigma_{1} .
\end{gathered}
$$

Используя эти формулы, после подстановки представления (4.2) в первое уравнение (2.2) получаем систему

$$
\begin{aligned}
-i \tau \sigma_{3}+\partial_{x} Z & =-i A_{3} \sigma_{3}-i\left(A_{1} \sigma_{1}+A_{2} \sigma_{2}\right) \Phi \\
-i \tau \Phi \sigma_{3}+\Phi \partial_{x} Z+\partial_{x} \Phi & =-i\left(A_{1} \sigma_{1}+A_{2} \sigma_{2}\right)-i A_{3} \sigma_{3} \Phi .
\end{aligned}
$$

Функции $A_{i}(x, t, u)$ в параметризации $\mathbf{S}=(\sin \theta \cos \varphi, \sin \theta \sin \varphi, \cos \theta)$ имеют вид

$$
\begin{aligned}
& A_{1}=-\tau^{-1}\left(w_{3} \cos \theta-\frac{p}{2}\right) w_{1} \sin \theta_{0}+w_{1} \tau^{-1} \sin \theta \cos \left(\varphi-\varphi_{+}\right)\left(w_{3} \cos \theta_{0}-\frac{p}{2}\right), \\
& A_{2}=w_{1} \sin \theta \sin \left(\varphi-\varphi_{+}\right), \\
& A_{3}=\tau^{-1}\left(w_{3} \cos \theta-\frac{p}{2}\right)\left(w_{3} \cos \theta_{0}-\frac{p}{2}\right)+w_{1}^{2} \tau^{-1} \sin \theta \cos \left(\varphi-\varphi_{+}\right) \sin \theta_{0} .
\end{aligned}
$$

Представим решение уравнений (4.4) и коэффициенты $A_{i}(x, t, u), \tau(u)$ в виде рядов по степеням $\left(u-u_{0}\right)$ :

$$
\begin{array}{rlrl}
\Phi & =\sum_{n=0}^{\infty} \Phi_{n}\left(u-u_{0}\right)^{n}, & Z=\sum_{n=0}^{\infty} Z_{n}\left(u-u_{0}\right)^{n}, \\
A_{i}=\sum_{n=-1}^{\infty} A_{i}^{(n)}\left(u-u_{0}\right)^{n}, & \tau=\sum_{n=-1}^{\infty} \tau^{(n)}\left(u-u_{0}\right)^{n} .
\end{array}
$$


Коэффициенты $\Phi_{n}, Z_{n}$ определяются цепочкой рекуррентных соотношений

$$
\begin{aligned}
& \partial_{x} \Phi_{n}-i \sum_{m=0}^{n+1} A_{3}^{(n-m)}\left[\Phi_{m}, \sigma_{3}\right]-i \sum_{m=0}^{n} \sum_{k=0}^{n+1} \Phi_{n-m}\left(A_{1}^{(m-k)} \sigma_{1}+A_{2}^{(m-k)} \sigma_{2}\right) \Phi_{k}+ \\
& \quad+i\left(A_{1}^{(n)} \sigma_{1}+A_{2}^{(n)} \sigma_{2}\right)=0 \\
& Z_{n}=i \sigma_{3} \int_{x}^{+\infty} d x^{\prime}\left(A_{3}^{(n)}-\tau^{(n)}\right)+i \int_{x}^{+\infty} d x^{\prime} \sum_{m=0}^{n+1}\left(A_{1}^{(n-m)} \sigma_{1}+A_{2}^{(n-m)} \sigma_{2}\right) \Phi_{m}
\end{aligned}
$$

где $n=0,1,2, \ldots, \Phi_{0}=\left(1+f_{3}\right)^{-1}\left(f_{1} \sigma_{1}+f_{2} \sigma_{2}\right) \sigma_{3}$,

$$
\begin{aligned}
& \left(\begin{array}{l}
f_{1} \\
f_{2} \\
f_{3}
\end{array}\right)=\left(\begin{array}{ccc}
\cos \theta_{0} & 0 & -\sin \theta_{0} \\
0 & 1 & 0 \\
\sin \theta_{0} & 0 & \cos \theta_{0}
\end{array}\right)\left(\begin{array}{l}
\widetilde{S}_{1} \\
\widetilde{S}_{2} \\
\widetilde{S}_{3}
\end{array}\right), \\
\widetilde{\mathbf{S}} & =\left(\sin \theta \cos \left(\varphi-\varphi_{+}\right), \sin \theta \sin \left(\varphi-\varphi_{+}\right), \cos \theta\right) .
\end{aligned}
$$

С помощью рядов для функции $\chi_{2}$, используя представление (4.1), находим разложение функции $\ln a^{*}\left(u^{*}\right)$ в ряд по степеням $\left(u-u_{0}\right)$ :

$$
\ln a^{*}\left(u^{*}\right)=\frac{i}{2} P-\frac{i \sqrt{k^{\prime}}}{\beta \sin \theta_{0}} H\left(u-u_{0}\right)+\cdots .
$$

Первые слагаемые содержат полевой импульс $P$ и энергию $H$ бризера. Под импульсом бризера мы понимаем разность между полным импульсом системы и импульсом волны намагниченности (2.1):

$$
P=\int_{-\infty}^{+\infty} d x\left(p \cos \theta_{0}-\partial_{x} \varphi \cos \theta\right)
$$

Аналогично определяем энергию бризера:

$$
H=\frac{1}{2} \int_{-\infty}^{+\infty} d x\left[\left(\partial_{x} \theta\right)^{2}+\left(\partial_{x} \varphi\right)^{2} \sin ^{2} \theta+\beta^{2} \sin ^{2} \theta-\left(p^{2}+\beta^{2}\right) \sin ^{2} \theta_{0}\right] .
$$

Используя соотношения $(2.24),(3.2)$ и явное выражение (2.28) для коэффициента $a^{*}\left(u^{*}\right)$, находим

$$
\begin{aligned}
& P=-2 i \ln a^{*}\left(u_{0}\right)=-2 i \ln \frac{\varphi^{*}\left(u_{0}\right)}{\varphi\left(u_{0}\right)}=-4 \arg \varphi\left(u_{0}\right) \quad(\bmod 2 \pi), \\
& H=\left.i \beta\left(k^{\prime}\right)^{-1 / 2} \sin \theta_{0} \frac{d \ln a^{*}\left(u^{*}\right)}{d u}\right|_{u=u_{0}}=-8 \operatorname{Im} \tau(\mu) .
\end{aligned}
$$

Видно, что импульс и энергия бризера существенно зависят от параметров $u_{0}, k^{\prime}$ волны намагниченности (2.1). Это обстоятельство следует учитывать при теоретическом описании физических свойств системы, образованной волной и солитонами. Кроме того, непосредственно из уравнений движения (1.1) следует еще один интеграл движения

$$
N_{0}=\int_{-\infty}^{+\infty} d x\left(S_{3}^{(0)}-S_{3}\right)=\int_{-\infty}^{+\infty} d x\left(\cos \theta_{0}-\cos \theta\right) .
$$


При квазиклассическом квантовании энергии солитонов интеграл движения $N_{0}$ определяет так называемое число спиновых отклонений [1]. Однако $N_{0}$ не содержится в семействе сохраняющихся величин (4.5). Для того чтобы найти явное выражение для $N_{0}$, заметим, что в точке $u=-i K^{\prime}+K / 2$ справедливы равенства

$$
\begin{gathered}
\varphi\left(-i K^{\prime}+\frac{K}{2}\right)=w_{1}\left(-i K^{\prime}+\frac{K}{2}\right)=0 \\
w_{3}\left(-i K^{\prime}+\frac{K}{2}\right)=-i \frac{\beta}{2}, \quad \tau\left(-i K^{\prime}+\frac{K}{2}\right)=-\frac{i \beta \cos \theta_{0}-p}{2} .
\end{gathered}
$$

При этом матричные функции $\chi_{1,2}^{(0)}, \Psi_{ \pm}$в точке $u=-i K^{\prime}+K / 2$ становятся диагональными. С учетом этих замечаний из первого уравнения (2.2) находим

$$
N_{0}=\left.\frac{2}{\beta} \ln \left[\Psi_{-}\left(x, t,-i K^{\prime}+\frac{K}{2}\right)\right]_{11}\right|_{x=-\infty} ^{x=+\infty}=\frac{4}{\beta} \ln \left|\frac{k^{2} \tilde{s} \tilde{c}}{(1+\tilde{d})\left(\tilde{d}-k^{\prime}\right)}\right| .
$$

\section{5. ВЗАИМОДЕЙСТВИЕ ДОМЕННОЙ ГРАНИЦЫ С ВОЛНОЙ НАМАГНИЧЕННОСТИ}

Решение модели (1.1), описывающее движение доменной стенки под действием нелинейной волны прецессии, получено в работе [4]. K сожалению, оно содержит неточности. Изложенный подход дает возможность заново рассмотреть задачу о взаимодействии волны прецессии (2.1) с доменной стенкой. В этом случае асимптотики волны должны иметь вид $\mathbf{S} \rightarrow \mathbf{S}^{( \pm)}$при $x \rightarrow \pm \infty$, где

$$
\mathbf{S}^{( \pm)}=\left(\sin \theta_{0} \cos \varphi_{ \pm}, \pm \sin \theta_{0} \sin \varphi_{ \pm}, \pm \cos \theta_{0}\right), \quad \varphi_{ \pm}=p x-\omega t+\delta_{ \pm},
$$

сдвиги фазы $\delta_{ \pm}$обусловлены типом доменной стенки.

Определим функции Йоста задачи о взаимодействии доменной стенки со спиновой волной как решения системы (2.2), имеющие асимптотическое поведение $\chi_{1,2} \rightarrow \chi_{1,2}^{(0)}$ при $x \rightarrow \mp \infty$. Здесь $\chi_{1,2}^{(0)}-$ точные решения системы $(2.2)$, соответствующие распределениям намагниченности (5.1):

$$
\chi_{1,2}^{(0)}=e^{ \pm i \sigma_{3} \varphi_{\mp} / 2}\left(\begin{array}{cc}
\beta(-u) & \pm \beta(u) \\
\mp \beta(u) & \beta(-u)
\end{array}\right) e^{\mp \tau \sigma_{3} \eta / i}, \quad \eta=x-\left(2 w_{3}+p \cos \theta_{0}\right) t .
$$

Как и ранее, множество $\Gamma=\{u: \operatorname{Im} \tau=0\}$ соответствует непрерывному спектру задачи рассеяния. На контуре $Г$ решения Йоста связаны между собой прежним соотношением (2.5), однако новые функции Йоста удовлетворяют ограничениям, которые несколько отличаются от (2.6):

$$
\begin{gathered}
\chi_{1,2}\left(-u+K+2 i K^{\prime}\right)=-\sigma_{3} \chi_{1,2}(u) \sigma_{3}, \quad \chi_{1,2}^{*}\left[\left(-u+2 i K^{\prime}\right)^{*}\right]= \pm i \sigma_{2} \chi_{1,2}(u), \\
\chi_{1,2}^{*}\left(u^{*}\right)=\frac{\left(1+k^{\prime}\right) \sqrt{k^{\prime}}}{2 \beta(u) \beta(-u)} \sigma_{2} \chi_{1,2}(u) \sigma_{2}, \quad u \in \Gamma
\end{gathered}
$$


Поэтому, хотя матрица перехода имеет прежнюю структуру (2.7), ее элементы удовлетворяют другим ограничениям:

$$
\begin{aligned}
a\left(-u+K+2 i K^{\prime}\right) & =a(u), & b\left(-u+K+2 i K^{\prime}\right) & =-b(u), \\
a^{*}\left[\left(-u+2 i K^{\prime}\right)^{*}\right] & =-a(u), & b^{*}\left[\left(-u+2 i K^{\prime}\right)^{*}\right] & =-b(u) .
\end{aligned}
$$

Введем функции $\chi_{+}(u)=\left(\chi_{1}^{(2)}(u), \chi_{2}^{(2)}(u)\right)$ и $\chi_{-}(u)=\left(\chi_{2}^{(1)}(u), \chi_{1}^{(1)}(u)\right)$, которые допускают аналитическое продолжение с контура $\Gamma$ в области $\Gamma_{+}$и $\Gamma_{-}$соответственно. На контуре $Г$ все базисные решения связаны между собой:

$$
\chi_{-}=\chi_{2} M_{+-}=\chi_{1} M_{--} i \sigma_{2}, \quad \chi_{+}=-\chi_{2} \sigma_{3} M_{++}=\chi_{1} M_{-+} \sigma_{1} .
$$

Здесь

$$
\begin{array}{ll}
M_{+-}=\left(\begin{array}{ll}
1 & a \\
0 & b
\end{array}\right), & M_{--}=\left(\begin{array}{cc}
1 & -a^{*} \\
0 & b
\end{array}\right), \\
M_{++}=\left(\begin{array}{ll}
b^{*} & 0 \\
a^{*} & 1
\end{array}\right), & M_{-+}=\left(\begin{array}{cc}
b^{*} & 0 \\
a & 1
\end{array}\right) .
\end{array}
$$

Связь решений Йоста на контуре Г эквивалентна условию сопряжения функций $\chi_{ \pm}(u)$ на том же контуре:

$$
\chi_{-}(u)=-\frac{1}{b^{*}\left(u^{*}\right)} \chi_{+}(u) \sigma_{3}\left(\begin{array}{cc}
1 & a(u) \\
a^{*}\left(u^{*}\right) & 1
\end{array}\right) .
$$

Используя равенства (5.4), находим

$$
b(u)=\frac{\operatorname{det} \chi_{-}(u)}{\operatorname{det} \chi_{2}^{(0)}(u)}, \quad b^{*}\left(u^{*}\right)=-\frac{\operatorname{det} \chi_{+}(u)}{\operatorname{det} \chi_{1}^{(0)}(u)} .
$$

Отсюда следует, что элементы матрицы перехода $b(u)$ и $b^{*}\left(u^{*}\right)$ аналитически продолжаются с контура $\Gamma$ в области $\Gamma_{-}$и $\Gamma_{+}$соответственно, за исключением, быть может, точек, где $\operatorname{det} \chi_{1,2}^{(0)}(u)=0$.

Нули коэффициентов $b(u)$ и $b^{*}\left(u^{*}\right)$ в областях $\Gamma_{-}$и $\Gamma_{+}$отвечают дискретному спектру задачи рассеяния. В силу редукций (5.3) у функции $b(u)$ имеются два особых нуля (2.9) и множество нулей (2.10). В данной задаче особые нули присутствуют всегда, так как они гарантируют наличие доменной стенки. Дополнительных нулей (2.10) может и не быть, поскольку они связаны с возбуждением бризеров на фоне нелинейной спиновой волны. Нули функции $b^{*}\left(u^{*}\right)$ комплексно сопряжены по отношению к нулям функции $b(u)$.

Для солитоноподобных возбуждений $a(u)=a^{*}\left(u^{*}\right) \equiv 0$, поэтому условие унимодулярности матрицы перехода приобретает простой вид $b(u) b^{*}\left(u^{*}\right)=1$. Для солитонного решения, которое описывает взаимодействие доменной стенки со спиновой волной в отсутствие бризеров, функция $b(u)$ оказывается мероморфной и легко восстанавливается по своим нулям и полюсам [10]:

$$
b(u)=\left.i \sigma(\operatorname{sn} v-i \operatorname{cn} v)(\operatorname{sn} w-i \operatorname{cn} w)\right|_{\substack{v=u+K / 2, w=-u+K / 2}}=-\frac{2 \sigma \beta(u) \beta(-u)}{\sqrt{k^{\prime}}\left(1+k^{\prime}\right)}, \quad \sigma= \pm 1 .
$$


Как и в работе [4], для построения интересующего нас решения модели (1.1) приме́ним метод "одевания" с той лишь разницей, что "одевать" будем не точное решение уравнения (1.1), а функцию $e^{-i \tau \sigma_{3} \eta}$, которая содержит все существенные особенности, необходимые для восстановления $U-V$ пары и решения $\chi_{-}(u)$. Решение $\chi_{-}(u)$ ищем в виде

$$
\chi_{-}(u)=\Psi(u) e^{-i \tau \sigma_{3} \eta}
$$

где $\Psi(u)$ - мероморфная двоякопериодическая функция с периодами $2 K, 4 i K^{\prime}$. Единственными особенностями функции $\Psi(u)$ в плоскости переменной $u$ являются полюсы в точках

$$
u= \pm\left(\frac{K}{2}+i K^{\prime}\right) \quad\left(\bmod \left(2 K, 4 i K^{\prime}\right)\right)
$$

Согласно формулам (5.2) матричная функция $\Psi(u)$ в области $\Gamma_{-}$должна удовлетворять редукциям

$$
\Psi\left(-u+K+2 i K^{\prime}\right)=-\sigma_{3} \Psi(u), \quad \Psi^{*}\left(\left(-u+2 i K^{\prime}\right)^{*}\right)=-i \sigma_{2} \Psi(u) \sigma_{3},
$$

а на контуре $\Gamma$ (при $\left.a=a^{*} \equiv 0\right)$ - условию

$$
\Psi^{\dagger}\left(u^{*}\right) \Psi(u)=\left[\beta(u) \beta^{*}\left(u^{*}\right)+\beta(-u) \beta^{*}\left(-u^{*}\right)\right] I .
$$

Для дальнейшего анализа полезно знать асимптотическое поведение функции $\chi_{-}(x, t, u)$ при $u \in \Gamma$ и $x \rightarrow \pm \infty$, которое нетрудно извлечь из (5.4):

$$
\begin{array}{ll}
\chi_{-} \rightarrow e^{i \sigma_{3} \varphi_{-} / 2}\left(\begin{array}{cc}
-\beta(u) b(u) & \beta(-u) \\
-\beta(-u) b(u) & -\beta(u)
\end{array}\right) e^{-\tau \sigma_{3} \eta / i}, & x \rightarrow-\infty \\
\chi_{-} \rightarrow e^{-i \sigma_{3} \varphi_{+} / 2}\left(\begin{array}{cc}
\beta(-u) & -\beta(u) b(u) \\
\beta(u) & \beta(-u) b(u)
\end{array}\right) e^{\tau \sigma_{3} \eta / i}, & x \rightarrow+\infty .
\end{array}
$$

Сопоставление формул (5.6) и (5.9) позволяет угадать зависимость от параметра $u$ элементов матрицы $\Psi$. Редукции (5.7), соотношение (5.8) и требование $\operatorname{det} \Psi=$ $b(u)\left[\beta^{2}(u)+\beta^{2}(-u)\right]$ полностью фиксируют алгебраическую структуру матричной функции $\Psi(u)$ :

$$
\Psi=\frac{1}{\sqrt{\gamma}}\left(\begin{array}{cc}
\beta(-u) a_{1}-\beta(u) b(u) a_{2} & -\beta(u) b(u) a_{1}+\beta(-u) a_{2} \\
\beta(u) a_{1}^{*}-\beta(-u) b(u) a_{2}^{*} & \beta(-u) b(u) a_{1}^{*}-\beta(u) a_{2}^{*}
\end{array}\right),
$$

где

$$
\gamma=\left|a_{1}\right|^{2}+\left|a_{2}\right|^{2}-\frac{\sigma \sin \theta_{0}}{\sqrt{1+\kappa^{2}}}\left(a_{1} a_{2}^{*}+a_{1}^{*} a_{2}\right) .
$$

При этом $\Psi^{-1}(u)=\Psi^{\dagger}\left[\beta(u) \beta^{*}\left(u^{*}\right)+\beta(-u) \beta^{*}\left(-u^{*}\right)\right]^{-1}$. 
Дополнительные дифференциальные ограничения на вид матрицы $\Psi$ состоят в том, что в области $\Gamma_{-}$функции $\partial_{x} \chi_{-} \chi_{-}^{-1}$ и $\partial_{t} \chi_{-} \chi_{-}^{-1}$ должны сводиться к выражениям следующего вида:

$$
\begin{aligned}
\partial_{x} \chi_{-} \chi_{-}^{-1}= & -i\left[w_{1}\left(S_{1} \sigma_{1}+S_{2} \sigma_{2}\right)+w_{3} S_{3} \sigma_{3}\right] \\
2 \partial_{t} \chi_{-} \chi_{-}^{-1}= & -i\left\{w_{1}\left(\left[\mathbf{S} \times \partial_{x} \mathbf{S}\right]_{1} \sigma_{1}+\left[\mathbf{S} \times \partial_{x} \mathbf{S}\right]_{2} \sigma_{2}\right)+\right. \\
& \left.+w_{3}\left[\mathbf{S} \times \partial_{x} \mathbf{S}\right]_{3} \sigma_{3}-2 w_{1}^{2} S_{3} \sigma_{3}-2 w_{1} w_{3}\left(S_{1} \sigma_{1}+S_{2} \sigma_{2}\right)\right\} .
\end{aligned}
$$

Можно показать, что левые части данных равенств, кроме слагаемых нужной алгебраической структуры с коэффициентами $w_{1}, w_{3}, w_{1} w_{3}$, содержат члены с “лишними" полюсами, а также слагаемые, не зависящие от спектрального параметра $u$. Требование отсутствия лишних слагаемых приводит к дифференциальным уравнениям для расчета функций $a_{1,2}(x, t)$, которые имеют простое решение

$$
a_{1,2}=\exp \left( \pm \frac{\beta}{2}\left(x \cos \theta_{0}+p t \sin ^{2} \theta_{0}+\Delta_{ \pm}\right) \mp \frac{i \varphi_{ \pm}}{2}\right),
$$

где $\varphi_{ \pm}=p x-\omega t+\delta_{ \pm}$, а $\delta_{ \pm}, \Delta_{ \pm}-$вещественные постоянные интегрирования. Если функции $a_{1,2}$ выбраны в соответствии с этими формулами, то решение Йоста $\chi_{-}$ имеет асимптотики (5.9). При этом из первого уравнения (5.10) находим искомые компоненты вектора $\mathbf{S}$ :

$$
\begin{aligned}
S_{3} & =\frac{1}{\gamma}\left[\left(\left|a_{1}\right|^{2}-\left|a_{2}\right|^{2}\right) \cos \theta_{0}+\frac{i \kappa \sigma \sin \theta_{0}}{\sqrt{\left(1+\kappa^{2}\right)}}\left(a_{1} a_{2}^{*}-a_{1}^{*} a_{2}\right)\right], \\
S_{1}-i S_{2} & =\frac{1}{\gamma}\left[\left(a_{1}^{2}+a_{2}^{2}\right) \sin \theta_{0}-\frac{2 a_{1} a_{2} \sigma}{\sqrt{\left(1+\kappa^{2}\right)}}\left(1-i \kappa \cos \theta_{0}\right)\right] .
\end{aligned}
$$

Формулы для $\mathbf{S}$, приведенные в работе [4], имеют неверный знаменатель, и потому не удовлетворяют уравнениям Ландау-Лифшица.

С математической точки зрения отличительная особенность рассмотренной задачи состоит в том, что матричная функция $\Psi(u)$ задачи Римана имеет двойные полюсы и не выражается через простые проекционные матрицы. Отметим также, что для доопределения задачи Римана не использовалось условие нормировки, было задано лишь асимптотическое поведение (5.10) функции $\chi$ - при $u \in \Gamma, x \rightarrow \pm \infty$.

Благодарности. Авторы признательны А. П. Танкееву за полезное обсуждение результатов работы и конструктивные замечания. Работа выполнена при поддержке фонда "Династия".

\section{Список литературы}

[1] А. М. Косевич, Б. А. Иванов, А. С. Ковалев, Нелинейные волны намагниченности. Динамические и топологические солитоны, Наукова думка, Киев, 1983.

[2] М. А. Шамсутдинов, И. Ю. Ломакина, В. Н. Назаров, А. Т. Харисов, Д. М. Шамсутдинов, Ферро- и антиферромагнитодинамика. Нелинейные колебания, волны и солитоны, Гилем, Уфа, 2007.

[3] А. В. Михайлов, А. И. Яремчук, Писъма в ЖЭТФ, 39:7 (1984), 296-298. 
[4] А. И. Яремчук, ТМФ, 62:1 (1985), 153-158.

[5] В.Е. Захаров, С.В. Манаков, С.П. Новиков, Л.П. Питаевский, Теория солитонов. Метод обратной задачи, Наука, М., 1980.

[6] Р.Ф. Бикбаев, А. И. Бобенко, А. Р. Итс, Уравнение Ландау-Лифиища. Теория точных решений. II, Препринт-Дон-ФТИ, Донецк, 1984.

[7] Р. Ф. Бикбаев, А. И. Бобенко, А.Р. Итс, Докл. АН СССР, 272:6 (1983), 1293-1298.

[8] Г. Бейтмен, А. Эрдейи, Высшие трансцендентные функиии. Т. 3. Эллиптические и автоморфные функиии. Функиии Ламе и Матъе, Наука, М., 1967.

[9] A. E. Borovik, S. Klama, S. I. Kulinch, Phys. D, 32:1 (1988), 107-134.

[10] Н. И. Ахиезер, Элементы теории эллиптических функиий, Наука, М., 1970.

[11] A. V. Mikhailov, "Integrable magnetic models", Solitons, Modern Problem in Condensed Matter Sci., 17, eds. S. E. Trullinger, V.E. Zakharov, V. L. Pokrovsky, Elsiver Sci., Amsterdam, 1986, 625-690.

[12] Yu. L. Rodin, Phys. D, 11:1-2 (1984), 90-108.

[13] А. Б. Борисов, В. В. Киселев, ТМФ, 54:2 (1983), 246-257.

Поступила в редакцию 1.10.2009 